$\mathrm{XIV}_{1} \cdot 3^{8} 3407$ (29. 10. 07). G. Sisson. Magnesium. carbonathydrat.

$\mathrm{XIV}_{1} \cdot 3^{8} 3466$ (3I.10.07). A. Gagon. Baryumcarbonat.

$\mathrm{XV}_{\mathrm{z}} \cdot 3^{8} 34^{26}$ (3o. Io. 07). E. J. Coudon. Elektrischer Apparat zum Erhitzen von Wasser.

Vom 23. I. 08 (angemeldet zwischen 9. und I5. I. O8):

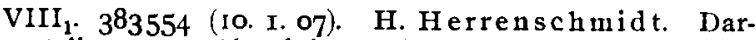
stellung von Aluminium.

$\mathrm{XII}_{6} \cdot 3^{8} 3540$ (3I. IO. 07). L. P. Basset. Element.

$\mathrm{XII}_{3} \cdot 3^{8} 3^{633}$ (5. II. O7). G. H. Noyau. Element.

$\mathrm{XIV}_{1} \cdot 3^{8} 353^{6}$ (3I. Io. 07). H. Coulon. Sauerstoff.

$\mathrm{XIV}_{1}$. $3^{83} 5^{85}$ (4. II. 07). E. Barbary. Ozonapparat zum Erzeugen von Ozon innerhalb einer Flüssigkeit.

$\mathrm{XIV}_{1} \cdot 3^{8} 3595$ (I2. I. O7). C. H. J a co b. Elektrolytische Darstellung von Acetaten, besonders Natriumacetat.

Vom 30. I. 08 (angemeldet zwischen 16. und 22. I. 08):

VIII. $_{12} 3^{8} 3_{57}^{8}$ (21. Io. 07). E. A. A. Grönwall, A. R. Lindblad und O.Stalhane. Elektrischer Schmelz. ofen.

$\mathrm{XIV}_{1} \cdot 3^{8375^{8}}$ (8. II. 07). C. E. Maistre. Baryumsulfat.
29. I. 08 ):

Vom 6. 2. o8 (angemeldet zwischen 23. und

VIII. 384072 (24. I. 07). A. M. G. Sebillot. Behandlung von kupferhaltigen Pyriten und Gewinnung sämtlicher Bestandteile.

VIII $_{2} \cdot 3^{8} 1_{187}$ (20. II.07). Centralstelle für wissenschaftich-technische Untersuchungen. Aluminiumlegierung.

$\mathrm{XII}_{5}$. 384035 (23. I. 07). M. Bouchet. Automatische Ladung von Akkumulatoren.

$\mathrm{XII}_{7}, 3_{4036}$ (16.11.07), F. Bergmann. Elektrischer Lichtbogenofen.

$\mathrm{XIV}_{1} \cdot 3^{8} 4006$ (4. I1. 07). H. S ch röder. Gewinnung von Erdalkaliphosphaten, Natrium-Aluminiumdoppelchloriden und Eisenchlorid aus Aluminium-EisenPhosphaten und Calcium-Aluminium-Ferraten.

$\mathrm{XIV}_{1}$. 384069 (16. Ir. 07). A. Sinding-Larsen. Behandlung von Aluminiumsilikat zur Gewinnung der Bestandteile.

$\mathrm{XIV}_{1} \cdot 3^{8}+\mathrm{II}_{3}(25$, I. 07). J.Steynis und H.Chaumat. Ozonisator.

$\mathrm{XIV}_{1} \cdot 3^{8}{ }_{144}$ (I8. II. 07). J. Hargreaves. Natriumsulfat.

$\mathrm{XIV}_{1} .8459$ (4. Il. 07; Zusatz zu 37I 797 vom 26. II. 06). O. Boeters und $R$. Wolffenstein. Konzentrieren von Salpetersäure mittels wasserbindender Nitrate.

\title{
SPRECHSAAL.
}

\section{Zur Elektroanalyse.}

Die Feststellungen des Herrn $T$ hiel in $Z$. f Elektroch. 14, 68 (Ig08, Nr. 6) nötigen wich, folgendes richtig zu stellen:

1. Ich habe bereits hervorgehoben (Z. f. Elektroch.14, 34), daß Herr Windelschmidt auf Grund seiner Untersuchungen nicht behaupten konnte, daß der fragliche schwarze Körper einen Kohlenstoffgehalt von $78 \%$ aufweise. Herr Thiel macht mich nun darauf aufmerksam, daß der höchste Koblenstoffgehalt bis zu $78 \%$ betragen habe. Dewgegenüber konstatiere ich, daß hier ein Maximun von $86 \%$ Kohlenstoff gefunden wurde. Bevor indes die Natur der fraglichen Substanz nicht erkannt und dieselbe rein isolierbar ist, was bisher nicht gelang, ist auch der letztere Wert nicht als sicher anzunebmen.

2. Herr Thiel behauptet, daß der durch Behandlung mit Kupferchlorid.Chlorammonium aus dem Oxalatniederschlage gewonnene schwarze Rückstand stets ebensoviel gewogen habe, wie das Oxalatnickel zu schwer war, und daß er gespannt sei, wie ich auf diese Tatsache hin die Unbrauchbarkeit der Isolierungsmethode erklären solle. Diese Erklärung wird Herrn Thiel sehr enttäuschen. Das Oxalatnickel enthält nämlich neben der fraglichen Kohlenstoffverbindung Ammoniunverbindungen (vorzugsweise Carbonat) in relativ großer Menge eingeschlossen, welche durch Auswaschen nicht zu entfernen sind ${ }^{1}$ ). So enthielten $50 \mathrm{~g}$ Oxalatnickel mehr als $0,2 \mathrm{~g}$ A mmoniumsalz, auf Chlorammonium berechnet, eingeschlossen. Nach dem Behandeln des Metalls mit Kupferchlorid-Chlorammonium findet sich (auch bei Reduktion von nur 0,2 $\mathrm{g}$ Metall) Chlorammonium in dem schwarzen Rückstande und ist durch Auswaschen (zuerst mit Salzsäure, dann mit Wasser bis zum Ausbleiben der Chlorreaktion) nicht vollständig zu entfernen ${ }^{2}$ ). Das Plus, welches sich bei der Bestimnung

I) Aehnliches beobachtete Clemens Winkler beim Ammoniakkobalt, nach der Ammoniakmethode nach Fresenius-Bergmann dargestellt.

2) Dieser Einschluß von Chlorammonium findet des Nickels aus dem oxalsauren Doppelsalz ergibt, setzt sich demnach zusammen aus der fraglichen organischen Substanz + Ammoniumsalz, und es ist allerdings wunderbar, daß Herr Windelschmidt das Gewicht dieses Rückstandes in Uebereinstimmung mit den Uebergewicht des Nickels fand. Abgesehen von dieser Tatsache, jst es jedem Analytiker, welcher sich mit der Bestimnung von Kohlenstoff in Metallen beschäftigt hat, bekannt, daß es unmöglich ist, einen fein verteilten kohligen Rückstand gewichtsanalytisch $z \mathfrak{z}$ bestimmen. Der Umstand, daß die Herren Thiel und Windelschnidt einen sehr schwankenden Kohlenstoffgehalt in dem fraglichen schwarzen Körper fanden (49 bis $78 \%$ !), mußte dieselben veranlassen, den Körper näher auf Reinheit zu untersuchen.

3. Ich habe mit meinen Bemerkungen in dieser Zeitschrift gegenüber Herrn Foerster gar nicht bezweckt, die Priorität bei Feststellung der Verbindung in Oxalatnickel für Aachen in Anspruch zu nehmen (die Sache ist doch viel zu unbedeutend); ich habe nich nur gegen den Wortlaut des Referates des Herrn Foerster gewandt. Da nun Herr Thiel von einer publizistischen Priorität spricht, die er beansprucht, so möchte ich zur Sache bemerken, daß Herr Dr. Verwer über den Kohlenstoffgehalt des Oxalatuickels schon in Jahre $190 \mathrm{I}$ in der Chemiker-Zeitung Mitteilung machte, und daß er kurz nachher (wie er mir kürzlich brieflich witteilte) hier weiter beobachtete, daß bei höheren Stromdichten nie kohlenstoff - freie Nickelniederschläge erzielt würden, selbst wenn die Elektrolyse lange vor totaler Ausscheidung des Metalls unterbrochen wurde. Der Nachfolger Verwers, Herr A. Fischer, hat dann zu einer späteren Zeit die Untersuchung wieder aufgenommen. Schließlich bemerke ich, daß Wolmann schon I897 in der Z. f. Elektroch. 3, 542 auf das zu hohe Gewicht des Oxalatnickels aufmerksam gemacht hat, ohne indes den Grund hierfür anzugeben. A achen, 16. Februar 1908.

A. Classen.

sich auch bei Anwendung von Kupferchlorid allein, an Stelle des Doppelsalzes. 
Herr Dr. F. A uerbach weist mich brieflich darauf hin, daß er vor vier Jahren in dieser Zeitschrift über eine Untersuchung des Doppelsalzes $\mathrm{MgCO}_{3} \cdot \mathrm{KHC \textrm {CO } _ { 3 }}$ berichtet hat, wie ich zu meinem Bedauern übersehen habe. In seiner Abhandlung teilt er auch gelegentlich mit, daßs die Störung der Gleichgewichte durch $\mathrm{CO}_{2}$ Abspaltung jedenfalls auch die Ursache davon ist, dals
Engel die Zusammensetzung der Lösungen verschieden fand, je nachdem er das Doppelsalz sich bilden oder sich zersetzen ließ. Wenn dieses sich auch aus seinen Versuchen folgern läßt, so hat Herr A u erbach jedoch die Bedeutung seiner Resultate für die fundamentale Frage der falschen Gleichgewichte nicht im geringsten betont.

E. H. B ü chner.
Zu den vorstehenden Zeilen möchte ich folgendes bemerken:

In der von Herrn Büchner übersehenen Abhandlung (Z. f. Elektroch. 10, I6r $-169\left[190_{4}\right]$ ) habe ich das System $\mathrm{MgCO}_{3}-\mathrm{KHCO}_{3}-\mathrm{H}_{2} \mathrm{O}$ bei drei Temperaturen eingehend untersucht und festgestellt, da $\beta$ es sich dabei um reversible Gleichgewichte handelt, deren scheinbare "Störungen" qualitativ mit der Phasenregel und quantitativ mit dem Massenwirkungsgesetz im Einklang sind Somit kamen für den vorliegenden Fall ,falsche Gleichgewichte" nicht mehr in Frage, und ich hatte keine Veranlassung, auf die irrige Annabme solcher durch Engel näher, als geschehen, einzugehen.

Charlottenburg, i7. Februar 1908.

Fr. Auerbach.

\section{BÜCHERSCHAU.}

Kongreßbericht des VI. Internationalen Kongresses für angewandte Chemle in Rom. 537 Originalabhandlungeu auf 6000 Seiten. Verlag von B. Löscher \& Co. in Rom. Preis der sieben Bände 6o Fr., des einzelnen Bandes I2 Mk.

Dem größten Teile der einzelnen Mitteilungen sind die Diskussionen beigefügt und beide sind in derjenigen Sprache zum Abdruck gebracht worden, in der sie gehalten wurden. Die dem Kongresse vorgelegten, aber nicht vorgetragenen Mitteilungen sind als Anhang zu den betreffenden Sektionsberichten zum Abdruck gekommen. Jeder Band enthält ein allgemeines Inhalts. verzeichnis des Bandes und am Ende jeder Sektion das Verzeichnis für diese, damit die einzelnen Sektionen für sich gebunden werden können. Der erste Teil des I. Bandes enthält einen Bericht über Organisation und Verlauf des Kongresses, mit den vier Hauptvorträgen von Ramsay, Moissan, Frank und Witt. Ferner enthält er organisatorische und geschäftliche Mitteilungen, sowie ein Autorenregister des Ganzen. Der $z$ weite Teil des I. Bandes umfaßt die Sitzungsberichte und die Mitteilungen der I. Sektion (analytische Chemie, Apparate und Instrumente) und der II. Sektion (anorganische Chemie und die entsprechenden Industrien). II. Band: Berichte der Sektion IIIA (Metallurgie und
Bergbau) und III B (Explosivstoffe), Berichte über den Stand und die Verhältnisse der Industrie der Explosivstoffe bei den verschiedenen Nationen, mit den entsprechenden Gesetzen in deren Staaten. III. Band Sektion IVA (Industrie der organischen Produkte), Sektion IV B (Farbstoffe und ihre Anwendungen), Sektion V (Industrie und Chemie des Zuckers). IV. Band: Sektion VIA (Fabrikation der Stärke und ihrer Derivate), Sektion VI B (Gärungsgewerbe mit besonderer Berücksichtigung der Weinbereitung) und Sektion VII (Agrikulturchemie). V. Band: Sek tion VIII A - B (Hygiene, medizinische und pharmazeutische Chemie) und Sektion VIII C (Bromatologie). VI. Band: Sektion IX (Photochemie, Photographie), Sektion X (physikalische Chemie, Elektrochemie) und Sektion XI (Rechts-und wirtschaftliche Fragen in Bezug auf die chemische Industrie). VII. Band: Berichte der drei auf den vorhergehenden Kongressen eingesetzten internationalen Kommissionen, nämlich: I. Die internationale Kommission für die Analyse der Kunstdünger und Futtermittel, 2. die internationale Analysenkommission, 3. die internationale Kommission für das Studium der Frage einheitlicher Untersuchungsmethoden der Nahrungsmittel. Diese Berichte waren bisher nicht im Buchbandel zu haben.

\section{HOCHSCHUL- UND PERSONAL-NACHRICHTEN.}

Bristol. Der Professor der Chemie am Clifton College A. Shenstone starb im Alter von $5^{8} \mathrm{Jahren}$. Karlsruhe. Prof. emer. Dr. G. Lunge-Zürich wurde die Würde eines Dr. ing. ehrenhalber verliehen.

Königsberg. Als o. Professor für Physik wurde Prof. Dr. W. Ka ufmann-Bonn berufen.

Krakau (Universität). Der Privatdozent der Pharmakognosie Dr. J. Lemberger ist gestorben.

\section{München (Universität).} A. Korn (Physik) ist auf seinen Wunsch vom Lehramte entboben worden.

Paris. Der Professor für Toxikologie A. Béhal wurde zum Professor für organische Chemie an der Ecole supérieure de Pharmacie der Universität ernanut.

\section{VEREINSNACHRICHTEN.}

\section{Deutsche Bunsen-Gesellschaft für angewandte physikalische Chemie.}

Anmeldungen zur Mitgliedschaft sind satzungsgemäß an den ersten Vorsitzenden, Herrn Geheimen Regierungsrat Prof. Dr. W. Nernst, Berlin IV. 35 . $\mathrm{K}$ arlsbad 26a, zu richten; die Anmeldungen müssen von einem Mitglied der Gesellschaft befürwortet sein.

\section{Adressenänderungen.}

Nr. 332. Elektrotechnische Gesellschaft Leipzig, jetzt: Leipzig, Schulstralie 3, Kaufmännisches Vereinshaus.

" 516. Frankenstein, jetzt: Stettin, Birkenallee 36.
Nr. 599. Pauli, jetzt: Frankfurt a. M.,, Feuerbach. Straße 48 .

" 936. Emmerich, jetzt: Homburg v. d. Höhe, Gluckenstein weg 5

"972. von Jüptner, jetzt: Wien IV, Victorgasse 2. 993. Stern, jetzt: Dr.

I070. Salm, jetzt: Berlin W., Geisbergstraße 23

, I I I4. Finkh, jetzt: Berlin SO. 33, Cuvry-Str. 2, II.

Nr. 185. Römer.
Gestorben. 